\title{
On the Albanese map of compact Kähler manifolds with numerically effective Ricci curvature
}

\author{
MiHAI PAUN
}

To the memory of Professor Michael Schneider

\section{Introduction.}

The following conjecture on compact Kähler manifolds with numerically effective Ricci class, is open since a few years (see [7]):

Conjecture 1. Let $X$ be a compact Kähler manifold with nef Ricci class. Then the Albanese map of $X$ is surjective.

(we refer to [6] for the definition and properties of nef line bundles on compact complex manifolds).

This conjecture was recently settled in the projective case by Qi Zhang (see [24]) using some characteristic $p$ methods. We also mention that for projective threefolds, Thomas Peternell and Fernando Serrano improved Zhang's result, and showed that the Albanese map is a submersion (see [21]).

The aim of the present paper is to give a differential-geometric approach of the problem; our main theorem solvs a particular case which will be specified below.

As it can be easily seen by the Aubin-Calabi-Yau theorem, the hypothesis on the Ricci class is equivalent to the existence of a sequence of Kähler metrics $\left(\omega_{k}\right)_{k>0}$ on $X$ such that

i) For each $k>0$, the metric $\omega_{k}$ belongs to a fixed cohomology class, say $\{\omega\}$.

ii) The Ricci curvature of $\omega_{k}$ is bounded from below by $-1 / k$. 
In this setting, our main result is stated as follows:

Theorem 1. Let $(X, \omega)$ be a compact complex manifold and let $\left(\omega_{k}\right)_{k}$ be a sequence of Kähler metrics on $X$ with the following properties:

i) for each $k>0$, the metric $\omega_{k}$ belongs to the cohomology class $\{\omega\}$.

ii) the Ricci curvature of $\omega_{k}$ is bounded from below by $-1 / k$.

iii) the diameters $d_{k}:=\operatorname{diam}\left(X, \omega_{k}\right)$ satisfy $d_{k} / \sqrt{k} \mapsto 0$ as $k$ goes to infinity.

Then the Albanese map of $X$ is surjective.

The proof of this result is given in the first part of the present paper; it rests on a theorem of Cheeger-Colding which we combine with a few ad-hoc Kähler arguments.

The main ideas are as follows: let us first suppose (for simplicity) that $X$ is a compact Riemannian manifold endowed with a metric $g$ of semipositive Ricci curvature. If $\beta$ denotes a harmonic 1 -form on $X$, the function $x \rightarrow|\beta|_{g, x}^{2}$ is constant (thanks to the Bochner formula, which implies the parallelism of the harmonic 1-forms). Now, in general, if the Ricci curvature is only bounded from below by $-1 / k$, we would like to estimate the variation of the function $x \rightarrow|\beta|_{k, x}^{2}$, on $X$. However, we can only get certain $L^{1}$ estimates, which in addition bring the diameter $d_{k}$ into the picture. The (undesired) hypothesis $d_{k} / \sqrt{k} \mapsto 0$ is needed to keep the $L^{1}$ norm of the variation of $x \rightarrow|\beta|_{k, x}^{2}$ close to zero, as $k$ goes to infinity.

In order to use these $L^{1}$ estimates, we associate to the sequence of Kähler metrics $\left(\omega_{k}\right)_{k}$ the subsets:

$$
\begin{aligned}
& \mathcal{D}\left(X,\left(\omega_{k}\right)_{k}\right):=\{p \in X / \text { there exists a neighbourhood } W \text { of } p \\
& \text { such that } \left.\lim _{k \rightarrow \infty} \operatorname{Vol}_{\omega_{k}}(W)=0\right\} \text {. } \\
& \mathcal{N D}\left(X,\left(\omega_{k}\right)_{k}\right):=X \backslash \mathcal{D}\left(X,\left(\omega_{k}\right)_{k}\right)
\end{aligned}
$$

Observe that the set $\mathcal{D}\left(X,\left(\omega_{k}\right)_{k}\right)$ can be actually very large -take for instance the projective plane $\mathbb{P}^{n}$ with the sequence of metrics

$$
\omega_{k}:=\frac{i}{2 \pi} \partial \bar{\partial} \log \left(\frac{1}{k}\left|z_{0}\right|^{2}+\left|z_{1}\right|^{2}+\cdots+\left|z_{n}\right|^{2}\right) .
$$

Then $\mathcal{D}\left(X,\left(\omega_{k}\right)_{k}\right)$ is $\mathbb{P}^{n}$ minus the point $[1 ; 0 \ldots ; 0]$. Nevertheless, by the Cheeger-Colding "Toponogov $L^{2}$ " theorem we will show that there exist at 
least one point in $\mathcal{N D}\left(X,\left(\omega_{k}\right)_{k}\right)$ which is of rank at least one for the Albanese map. Finally, we show that such a point is automatically of maximal rank, so $\alpha_{X}$ is surjective (in the proof of this final step, we use a Sobolev-type inequality due to S. Gallot, see [11]).

In the second part we establish an analytical setting such that the conditions i)-iii) of the theorem 1 are satisfied. In a few words this can be formulated as follows.

Let $\{\alpha\}$ be a nef cohomology class (see [5]). We first show that the existence of a closed positive current $T \in\{\alpha\}$ whose potential $f$ is such that $\exp (-f)$ is integrable provides the class $\{\alpha\}$ with a sequence of smooth representatives $\alpha_{k}$, almost positive, for whith we have a uniform control of the integrals $\int_{X} \exp \left(-f_{k}\right)$ associated with their potentials (see proposition 2.1 for the precise statement). Then we use exactly this potentials to solve a Aubin-Calabi-Yau equation, and in this way we get a sequence of Kähler metrics on $X$, which satisfy the assumptions $i$ )-ii) of theorem 1 ; in addition, the volume of certain geodesic balls of radius 1 of $\left(X, \omega_{k}\right)$ is uniformly bounded from below. On the other hand we obtain an upper bound of the volume of these balls in terms of the Ricci curvature, global volume and diameter (see lemma 2.3). As the total volume is constant, we get an upper bound for the sequence of diameters.

In this way, we obtain the following result:

Theorem 2. Let $X$ be a compact Kähler manifold such that the Ricci class of $X$ is numerically effective and integrable. Then $X$ has a sequence of metrics with the properties i)-iii) of the theorem 1.

(actually, under the assumptions of the theorem 2 we obtain a uniform upper bound for $d_{k}$ ).

In the final part of this paper, we get some results also in the case that the sequence of diameters $\left(d_{k}\right)_{k}$ goes to infinity.

Actually, if $d_{k} \mapsto \infty$ we observe (lemma 2.3) that the dimension of the Gromov-Hausdorff limit of $\left(X, \omega_{k}\right)_{k}$ cannot be maximal (i.e. this sequence collapses in the terminology of Cheeger-Colding). If in addition we suppose that the collapsing occurs only in the fibers of $\alpha_{X}$, then we show that the irregularity of $X$ is strictly less than its complex dimension (in general, for a compact Kähler manifold with nef Ricci curvature, the irregularity is less then the complex dimension, see [19]). The exact statement is:

Theorem 3. Let $\left(X, \omega_{k}\right)_{k}$ be a compact complex manifold endowed with a sequence of Kähler metrics such that: 
i) Each metric $\omega_{k}$ belongs to a fixed cohomology class and $\operatorname{Ricci}_{\omega_{k}} \geq$ $-1 / k \omega_{k}$.

ii) There exists a constant $C>0$ and a sequence of positive numbers $\left(C_{k}\right)_{k}$ such that $\lim _{k \rightarrow \infty} C_{k} / \sqrt{k}=0$ and for all $\gamma$ in $H_{1}(X, \mathbb{Z})$ modulo torsion we have:

$$
C \leq|\gamma|_{k, p} \leq C_{k} l_{\text {alg }}(\gamma)
$$

for all $p \in X$.

Then, if $d_{k}:=\operatorname{diam}\left(X, \omega_{k}\right)$ tends to infinity, the irregularity of $X$ is bounded by $n-1$.

Acknowledgements. It is an honour and a pleasure to thank Gérard Besson, Jean-Pierre Demailly, Sylvestre Gallot and Paul Gauduchon for their carefull reading and valuable suggestions concerning this paper.

\section{1.}

The proof of theorem 1 will occupies the whole first part.

\section{Proof of the theorem 1.}

To begin with, let us fix the notation. We denote by $\Delta_{k}$ the Laplace-Beltrami operator of the metric $\omega_{k}$ acting on 1 -forms, by $|\cdot|_{k}$ the norm on $\Lambda^{*} T_{X}^{*}$ given by $\omega_{k}$ and by $\nabla_{k}$ the Levi-Civita connexion of $\omega_{k}$.

Then for every 1 -form $\beta$ one has the Bochner formula

$$
\Delta_{k}(\beta)=\nabla_{k}^{*} \nabla_{k} \beta+\rho_{k}(\beta)
$$

where the eigenvalues of the (linear) operator $\rho_{k}$ are the same as those of the Ricci curvature of $\omega_{k}$, hence bounded from below by $-1 / k$. The metric $\omega_{k}$ is Kähler, so for all holomorphic 1 -forms $\beta$ we have $\Delta_{k}(\beta)=0$. In conclusion, for all holomorphic 1 -form $\beta$, by integrating the Bochner formula (1) we get

$$
\int_{x \in X}\left|\nabla_{k} \beta\right|_{k, x}^{2} d V_{\omega_{k}}(x) \leq \frac{1}{k}\|\beta\|_{L^{2}\left(X, \omega_{k}\right)}^{2} .
$$

By the Bochner formula we thus only get a bound of the $L^{2}$ norm of the covariant derivative of $\beta$. In these conditions, it seems difficult to control the pointwise difference $\left.|| \beta\right|_{k, x} ^{2}-|\beta|_{k, y}^{2} \mid$ on $X \times X$; the best one can hope is 
to get a $L^{1}$ estimates of this term. This is actually what we get, thanks to the following "Toponogov $L^{2}$ " theorem of Cheeger-Colding.

The result we need (see [1] and equally [12]) is:

Theorem (Cheeger-Colding). Let $(M, g)$ be a m-dimensional compact Riemannian manifold, such that $\operatorname{Ricci}_{g} \geq-(m-1) \delta g(\delta \geq 0)$. Consider the geodesic ball $B_{g}(p, R)$ of radius $R \leq 1 / \sqrt{\delta}$. Then for every function $f \in L^{2}(M)$ we have

$$
\begin{aligned}
\frac{1}{\operatorname{Vol}_{g}\left(B_{g}(p, R)\right)^{2}} \int_{B_{g}(p, R) \times B_{g}(p, R)} & \left(\int_{\gamma_{x y}} f^{2}\right) d V_{g}(x) d V_{g}(y) \leq \\
& \leq C(m) V_{\delta}(R, 2 R)^{-1} R\|f\|_{L^{2}\left(B_{g}(p, 2 R)\right)}^{2}
\end{aligned}
$$

The notation used is the following: for almost every pair of points $(x, y)$ of the ball $B_{g}(p, R)$ we have a unique geodesic between them; we denote it by $\gamma_{x y}$. In a Riemannian space of constant curvature $-\delta$ we denote by $\operatorname{Vol}\left(B_{\delta}(R)\right)$ the volume of the geodesic ball of radius $R$ and we write $V_{\delta}(R, 2 R):=\frac{\operatorname{Vol} B_{\delta}(R)}{\operatorname{Vol} B_{\delta}(2 R)}$. Finally, we denote

$$
\|f\|_{L^{2}\left(B_{g}(p, 2 R)\right)}^{2}:=\frac{1}{\operatorname{Vol}_{g}\left(B_{g}(p, 2 R)\right)} \int_{B_{g}(p, 2 R)} f^{2}(x) d V_{g}(x) .
$$

Now let $x$ and $y$ be two points of the manifold $\left(X, \omega_{k}\right)$ such that there exist a unique geodesic $\gamma_{k, x y}$ between them (note that this property is satisfied for almost all $x$ and $y$ ). Then

$$
\left.|| \beta\right|_{k, x} ^{2}-|\beta|_{k, y}^{2}|=| \int_{0}^{d_{k}(x, y)} d / d t\left(|\beta|_{\gamma_{k, x y}(t)}^{2}\right) d t\left|\leq 2 \int_{\gamma_{k, x y}}\right|\left\langle\nabla_{k} \beta, \beta\right\rangle \mid .
$$

We now apply the theorem of Cheeger-Colding with the following data: the manifold $(M, g):=\left(X, \omega_{k}\right), R:=d_{k}, \delta:=1 / k, f^{2}(x):=\left|\left\langle\nabla_{k} \beta, \beta\right\rangle\right|_{k, x}$. Recall that

$$
V_{1 / k}\left(d_{k}, 2 d_{k}\right)^{-1}=\frac{\int_{0}^{2 d_{k} / \sqrt{k}} s h^{2 n-1}(t) d t}{\int_{0}^{d_{k} / \sqrt{k}} s h^{2 n-1}(t) d t} .
$$

By hypothesis iii) relative to the behavior of the sequence of diameters $d_{k}$ as $k$ goes to infinity, we can assume that quantity (4) is uniformly bounded 
from above with respect to $k$ (because the limit of (4) at infinity is $4^{n}$ ). By the theorem of Cheeger-Colding, we get the inequality

$$
\begin{aligned}
& \left.\frac{1}{\operatorname{Vol}_{k}(X)^{2}} \int_{X \times X}|| \beta\right|_{k, x} ^{2}-|\beta|_{k, y}^{2} \mid d V_{k}(x) d V_{k}(y) \leq \\
& \leq C^{\prime}(n) \frac{d_{k}}{\operatorname{Vol}_{k}(X)} \int_{X}\left|\left\langle\nabla_{k} \beta, \beta\right\rangle\right|_{x} d V_{k}(x)
\end{aligned}
$$

Next, by the Cauchy inequality and the Bochner formula (2), we obtain

$$
\begin{aligned}
\int_{X}\left|\left\langle\nabla_{k} \beta, \beta\right\rangle\right|_{k, x} d V_{k}(x) & \leq\left(\int_{X}\left|\nabla_{k} \beta\right|_{k, x}^{2} d V_{k}(x) \int_{X}|\beta|_{k, x}^{2} d V_{k}(x)\right)^{1 / 2} \leq \\
& \leq \frac{1}{\sqrt{k}} \int_{X}|\beta|_{k, x}^{2} d V_{k}(x) .
\end{aligned}
$$

On the other hand, we have a certain uniformity with respect to $k$ thanks to the hypothesis $\omega_{k} \in\{\omega\}$. Actually this implies first that $\operatorname{Vol}_{k}(X)=$ $\operatorname{Vol}(X, \omega)$, and secondly

$$
\int_{X}|\beta|_{x, k}^{2} d V_{\omega_{k}}(x)=i \int_{X} \beta \wedge \bar{\beta} \wedge \omega_{k}^{n-1}=i \int_{X} \beta \wedge \bar{\beta} \wedge \omega^{n-1}:=\|\beta\|_{L^{2}}^{2} ;
$$

so the $L^{2}\left(X, \omega_{k}\right)$ global norm of each holomorphic 1 -form is a cohomological quantity, which in our case does not depend on $k$.

In conclusion, we get

$$
\left.\frac{1}{\operatorname{Vol}(X)} \int_{X}|| \beta\right|_{k, x} ^{2}-\frac{1}{\operatorname{Vol}(X)}\|\beta\|_{L^{2}}^{2} \mid d V_{k}(x) \leq C^{\prime}(n) d_{k} / \sqrt{k}\|\beta\|_{L^{2}}^{2} .
$$

Let us denote $\delta_{k}:=\sqrt{d_{k} / \sqrt{k}}$. Inequality (5) implies the existence of an open set $\Lambda_{k}(\beta) \subset X$ such that

1) the volume of $\Lambda_{k}(\beta)$ satisfies $\operatorname{Vol}_{\omega_{k}}\left(\Lambda_{k}(\beta)\right) \geq\left(1-\delta_{k}\right) \operatorname{Vol}(X)$

2) for all $p \in \Lambda_{k}(\beta)$ we have

$$
\left.|| \beta\right|_{k, p} ^{2}-\frac{1}{\operatorname{Vol}(X)}\|\beta\|_{L^{2}}^{2} \mid \leq C(n) \delta_{k}\|\beta\|_{L^{2}}^{2} .
$$

Recall that by hypothesis iii), the sequence $\delta_{k}$ goes to zero. Hence there exists a certain rank $k_{0}$ (which does not depend on $\beta$ ) such that for $k \geq k_{0}$ we have 
$\left.2^{\prime}\right)$ For all $p \in \Lambda_{k}(\beta)$ the global $L^{2}$-norm of $\beta$ verifies $\|\beta\|_{L^{2}}^{2} \leq$ $2 \operatorname{Vol}(X)|\beta|_{k, p}^{2}$.

In order to use these estimates, we consider the subsets of $X$

$$
\begin{gathered}
\mathcal{N D}\left(X,\left(\omega_{k}\right)_{k}\right):=\{p \in X / \text { for all neighbourhood } V \text { of } p \text { we have } \\
\left.\quad \operatorname{Vol}_{\omega_{k_{m}}}(V)>C(V)>0 \text { for a sub-sequence }\left(k_{m}\right)_{m}\right\} \\
\mathcal{D}\left(X,\left(\omega_{k}\right)_{k}\right):=\left\{\begin{aligned}
p \in X / \text { there exists a neighbourhood } W \text { of } p \\
\text { such that } \left.\lim _{k \rightarrow \infty} \operatorname{Vol}_{\omega_{k}}(W)=0\right\} .
\end{aligned}\right.
\end{gathered}
$$

It is obvious that $X=\mathcal{N} \mathcal{D}\left(X,\left(\omega_{k}\right)_{k}\right) \bigcup \mathcal{D}\left(X,\left(\omega_{k}\right)_{k}\right)$ and that this is a disjoint union (see remark 4 in the second part for some properties of these subsets of $X$ in the case that the metrics are given by a Monge-Ampère equation).

We next show that if $q(X) \neq 0$, the set $\mathcal{N D}\left(X,\left(\omega_{k}\right)_{k}\right)$ contains at least one point which is not of rank zero for the Albanese map $\alpha_{X}$.

As the metrics $\left(\omega_{k}\right)_{k}$ are all in the same cohomology class, we have

$$
\int_{X} \omega \wedge \omega_{k}^{n-1}=\operatorname{Vol}(X)
$$

so if we denote by $\lambda_{j}^{k}(x)$ the eigenvalues of $\omega_{k}$ at $x$ with respect to $\omega$, the equality (6) can be written as

$$
\int_{X} \sum_{j=1}^{n} \frac{1}{\lambda_{j}^{k}(x)} d V_{\omega_{k}}=n \operatorname{Vol}(X) .
$$

By using again the fact that the volume of the manifold $X$ with respect to $\omega_{k}$ is constant, the relation (7) implies the existence of a sequence of open subsets $\left(U_{k}\right)_{k} \subset X$ such that for each positive $k$ we have $\operatorname{Vol}_{\omega_{k}}\left(U_{k}\right) \geq$ $\frac{1}{2} \operatorname{Vol}(X)$ and $\sum_{j=1}^{n} \frac{1}{\lambda_{j}^{k}(p)} \leq 2 n$ for all $p \in U_{k}$. So for each point $p \in U_{k}$ we get $\lambda_{j}^{k}(p) \geq \frac{1}{2 n}$.

Given an arbitrary holomorphic 1 -form $\beta$, let us consider the sequence $U_{k}(\beta):=U_{k} \cap \Lambda_{k}(\beta)$. Then for all integers $k$ greater than a certain rank $k_{1}$ (which does not depend on $\beta$ ) we have $\operatorname{Vol}_{\omega_{k}}\left(U_{k}(\beta)\right) \geq \frac{1}{4} \operatorname{Vol}(X)$ (by property 1 of $\left.\Lambda_{k}(\beta)\right)$. If we denote $k_{2}:=\max \left(k_{0}, k_{1}\right)$ we observe that

$$
\overline{\bigcup_{k \geq k_{2}} U_{k}(\beta)} \cap \mathcal{N D}\left(X,\left(\omega_{k}\right)_{k}\right) \neq \emptyset
$$


because otherwise $\overline{\bigcup_{k \geq k_{2}} U_{k}(\beta)}$ is a subset of $\mathcal{D}\left(X,\left(\omega_{k}\right)_{k}\right)$ and thanks to the compactness of $X$, we would have

$$
\lim _{k \rightarrow \infty} \operatorname{Vol}_{\omega_{k}}\left(\overline{\bigcup_{m \geq k_{2}} U_{m}(\beta)}\right)=0 .
$$

But on the other hand $\operatorname{Vol}_{\omega_{k}}\left(\overline{\bigcup_{m \geq k_{2}} U_{m}(\beta)}\right) \geq \operatorname{Vol}_{\omega_{k}}\left(U_{k}(\beta)\right) \geq 1 / 4 \operatorname{Vol}(X)$ for all $k \geq k_{2}$, contradiction.

So we can pick a point $q$ in the intersection in (8). Then we get a sequence of points $q_{m} \in U_{k_{m}}(\beta)$ which converges to $q$. From relation $\left(2^{\prime}\right)$ and the fact that at all points of $U_{k}$ the eigenvalues $\lambda_{j}^{k}$ are bounded from below by $1 / 2 n$, we infer that

$$
\|\beta\|_{L^{2}} \leq 2 \operatorname{Vol}(X)|\beta|_{k_{m}, q_{m}} \leq 3 n \operatorname{Vol}(X)|\beta|_{q_{m}} .
$$

When $m$ goes to infinity, we get

$$
\|\beta\|_{L^{2}} \leq 3 n \operatorname{Vol}(X) \sup _{z \in \mathcal{N} \mathcal{D}\left(X,\left(\omega_{k}\right)_{k}\right)}|\beta|_{1, z}
$$

For the rest of the proof, we may assume the existence of some nonidentically zero holomorphic 1 -form on $X$ (otherwise the surjectivity of the Albanese map it is not very hard to prove). Then by inequality (9) we get that the set $\left.\mathcal{N D}\left(X, \omega_{k}\right)_{k}\right)$ contains at least one point $p_{0}$ which is not of rank 0 for $\alpha_{X}$.

We will see in proposition 1.3 below that this property of $p_{0}$ implies the local non-degeneracy at $p_{0}$ of the sequence of metrics, transversally to the fibers of the Albanese map. To be precise, we need the following definition:

Definition 1.1. The sequence of metrics $\left(\omega_{k}\right)_{k}$ is called non-degenerate locally at $p_{0}$ on the transverse directions to the fibers of the Albanese map if there exists a neighborhood $U$ of $p_{0}$ and a constant $C>0$ such that for every point $p \in U$ and every vector $v \in T_{p} X$, orthogonal to $\operatorname{ker}\left(d \alpha_{X, p}\right)$ with respect to the metric $\omega$, we have

$$
|v|_{\omega_{k}} \geq C|v|_{\omega} .
$$

It is easy to see that this non-degeneracy property imply the following assertion: for every global holomorphic 1 -form $\beta$, every $p \in U$ and every $k \geq 1$ we have

$$
|\beta|_{k, p} \leq C|\beta|_{1, p}
$$


Actually, for any point point $p \in U$ we denote by $H_{p}$ the subspace of the holomorphic tangent space at $p$, equal to the orthogonal (with respect to $\omega$ ) of the kernel of the differential of $\alpha_{X}$ at $p$. We can take for each positive $k$ certain local coordinates on $p$, namely $z_{1, k}, \ldots, z_{n, k}$, such that the first $m=m(p)$ vectors $\left(\frac{\partial}{\partial z_{j, k}}\right)$ yield an orthonormal basis with respect to $\omega$ and orthogonal to the space $H_{p}$ with respect to $\omega_{k}$, and such that the other vectors $\frac{\partial}{\partial z_{m+j, k}}$ sits on the kernel of $d \alpha_{X, p}$. The local non-degeneracy of the sequence of metrics implies

$$
\lambda_{j}^{k}(p)=\left|\frac{\partial}{\partial z_{k, j}}\right|^{2} \geq C_{1}
$$

for all $j=1 \ldots m$ and $p \in U$. On the other hand, each global holomorphic 1 -form $\beta$ can be written at $p$ as

$$
\beta_{p}=\sum_{j=1}^{m} \beta_{j, k} d z_{j, k},
$$

and as for $j=1 \ldots m$ the eigenvalues $\lambda_{j}^{k}(p)$ are bounded from below by a constant $C_{1}$ we get:

$$
|\beta|_{k, p} \leq C_{1}^{-1 / 2}|\beta|_{p}
$$

Therefore, modulo proposition 1.3 (which will be proved at the end of this section), we have the corollary:

Corollary 1.2. For each holomorphic 1-form $\beta$, there exists a sequence of open subsets in $X$ namely $\Lambda_{k}(\beta) \subset X$, such that:

1) for all positive $k$ we have $\operatorname{Vol}_{\omega_{k}}\left(\Lambda_{k}(\beta)\right) \geq\left(1-\delta_{k}\right) \operatorname{Vol}(X)$.

2) for all $p \in \Lambda_{k}(\beta) \cap U$ we have

$$
\|\beta\|_{L^{2}}^{2} \leq C|\beta|_{p}^{2}
$$

if $k \geq k_{2}$.

If the Albanese map $\alpha_{X}$ were not surjective, there would exist a non identically zero holomorphic 1 -form $\beta$, such that $\beta_{p_{0}}=0$. Let us consider the sequence $\Lambda_{k}(\beta)$ (corresponding to this 1-form) given by corollary 1.2 above. The fact that $p_{0}$ belongs to the set $\mathcal{N D}\left(X,\left(\omega_{k}\right)_{k}\right)$, shows the existence of a sequence of points $p_{m} \in \Lambda_{k_{m}}(\beta) \cap U$ convergent to $p_{0}$. Actually, we can 
consider a decreasing sequence $\left(U_{m}\right)_{m}$ of neighborhoods of $p_{0}$ such that their intersection is exactly $p_{0}$. As $p \in \mathcal{N D}\left(X,\left(\omega_{k}\right)_{k}\right)$, for each $m$ we have $\operatorname{Vol}_{\omega_{k}}\left(U_{m}\right) \geq c(m)>0$ if $k$ belongs to a non-bounded sequence of positive integers. By the first part of the corollary 2. 1, we get a rank $k_{m} \geq k_{2}$ such that $\Lambda_{k_{m}}(\beta) \cap U_{m} \neq \emptyset$. So we can take a point $p_{m}$ in the intersection, and we select such a point arbitrarily.

Now, by the second part of corollary 2.1 we get

$$
\|\beta\|_{L^{2}}^{2} \leq C|\beta|_{1, p_{m}}^{2} .
$$

As $m$ goes to infinity, the right hand side of this inequality tends to zero. This implies $\beta=0$, contradiction.

We will now prove the result needed in the proof of the previous theorem.

Proposition 1.3. Under the hypotheses of theorem 1, the sequence $\left(\omega_{k}\right)_{k}$ is non-degenerate locally on the transverse directions to the fibers of the Albanese map of $X$ at each point $p_{0}$ which is not of rank zero for the differential of $\alpha_{X}$.

Proof. The proof presented here owes a lot to the remarks of S. Gallot, who suggested a substantial simplification of our previous arguments.

We start by an observation, which is a consequence of Gallot's Sobolev inequality. Under the hypotheses of the proposition, for all holomorphic 1 -forms $\beta$ and all $k \geq 1$ we have

$$
|\beta|_{k, p} \leq C\|\beta\|_{L^{2}}
$$

for all $p \in X$, where $C$ is a constant which does not depend on the sequence of metrics. To see this, we remark first of all that, by Kato's inequality (see for instance [15]) and the bound for the Ricci curvature, we have

$$
|\beta|_{k, p} \Delta_{k}\left(|\beta|_{k, p}\right) \leq \operatorname{Re}\left\langle\nabla_{k}^{*} \nabla_{k} \beta, \beta\right\rangle_{k, x} \leq \frac{1}{k}|\beta|_{k, x}^{2} .
$$

Therefore we have $\Delta_{k}\left(|\beta|_{k, p}\right) \leq \frac{1}{k}|\beta|_{k, p}$ for all positive integer $k$ and all $p \in X$. We recall here $\mathrm{S}$. Gallot's result (see [11]):

Theorem (Gallot). Let $(M, g)$ be a compact Riemannian manifold of dimension $m$, such that $\operatorname{Ricci}_{g} \geq-(m-1) \delta^{2} g, \operatorname{Vol}_{g}(M)=V$ and 
$\operatorname{diam}(M, g) \leq D$. Then for every positive function $f$ which satisfies $\Delta(f) \leq \delta^{2} f$ almost everywhere on $M$, we have the estimate

$$
|f|_{L^{\infty}} \leq \frac{1}{V}\left(1+\eta_{D}(\delta)\right)\|f\|_{L^{2}}
$$

where $\eta_{D}$ is a function uniformly bounded in terms of $D \delta$.

So by Kato and Gallot's results applied to the Riemannian manifold $\left(X, \omega_{k}\right)$, we get immediately the inequality (12), with a constant $C$ equal for instance to $2 / \operatorname{Vol}(X)$, if $k \gg 0$.

Let $p_{0}$ be a point which is not of rank zero for $\alpha_{X}$; let $U$ be a neighborhood of $p_{0}$ which does not intersect the (analytic) set of points where the rank of $\alpha_{X}$ is equal to zero. With the previous notation, for all $v \in H_{p}$ such that $|v|_{\omega}=1$ we have $\left|d \alpha_{X, p}(v)\right| \geq \delta$ for some constant $\delta=\delta(U)>0$. By inequality (12), we get $\left|d \alpha_{X}(v)\right| \leq C|v|_{k}$ for some (other) constant $C$. In conclusion, for all vector $v \in H_{p}$, of norm equal to 1 with respect to $\omega$ we have

$$
|v|_{k} \geq C
$$

where the constant $C$ is uniform with respect to $k$ and $p \in U$. The proposition is proved.

\section{Remark.}

1) Of course, one can also apply the theorem of Cheeger-Colding in a local manner; in this way one obtains the estimate

$$
\left.\int_{X}|| \beta\right|_{k, p} ^{2}-\left\||\beta|_{k}\right\|_{L^{2}\left(B_{k}(p, r)\right)}^{2} \mid d V_{\omega_{k}} \leq C(n) \frac{r}{\sqrt{k}}\|\beta\|_{L^{2}}^{2}
$$

(without any hypothesis on the sequence of diameters).

To use this type of information, it is necessary to obtain some uniform estimates for the sequence of numbers $\left\||\beta|_{k}\right\|_{L^{2}\left(B_{k}(p, r)\right)}^{2}$, where the radius $r$ must be chosen in order to ensure the convergence to zero of the right hand side of (14). We have seen that this sequence is constant for $r=d_{k}$, but in general it seems difficult to control the quotient $d_{k} / \sqrt{k}$.

\section{2.}

In this section our aim is to derive an analytic setting which guarantees the validity of hypothesis $i$ )-iii) in theorem 1. 
Let $\{\alpha\}$ be a numerically effective $(1,1)$-cohomology class (see [5]). Then by definition we have representatives $\alpha+i \partial \bar{\partial} f_{k}$ bounded from below by $-1 / k \omega$ (pointwise), and such that $\max _{X}\left(f_{k}\right)=0$. It is very useful to control the behavior of the $L^{1}$ norm of the functions $\exp \left(-f_{k}\right)$ as $k$ goes to infinity. Although in general it seems difficult to get such information, we will indicate some particular cases for which we can do this.

The type of hypothesis we will consider here is:

Definition. We say that a $(1,1)$ class $\{\alpha\}$ is integrable if it contains a closed positive current $T$ of the form

$$
T=\alpha+i \partial \bar{\partial} \varphi
$$

with $\int_{X} \exp (-\varphi) d V<\infty$.

We then get the following result.

Proposition 2.1. Let $\{\alpha\}$ be a numerically effective and integrable $(1,1)$ cohomology class on $X$. Then there exists a positive constant $C>0$ such that for all $k>0$ there exists a function $f_{k} \in \mathcal{C}^{\infty}(X)$ such that:

i) $f_{k}(x) \leq 0$ for all $x \in X$.

ii) $\alpha+i \partial \bar{\partial} f_{k} \geq-1 / k \omega$.

iii) $\int_{X} \exp \left(-f_{k}\right) d V \leq C$.

Before giving the proof of this result, let us indicate some examples of nef cohomology classes which are integrable.

\section{Examples.}

1) All semi positive $(1,1)$-classes are integrable.

2) More generally, this property is satisfied for every cohomology class $\{\alpha\}$ which contains a closed positive current whose Lelong numbers are small enough, i.e. $\max _{X} \nu(T, x)<2$. In fact, this is a consequence of the following lemma, due to H. Skoda (see [22]).

Lemma (Skoda). Let $\phi$ be a psh function on a open set of $\mathbb{C}^{n}$. If $\nu(\phi, x)<$ 1 then the function $\exp (-2 \phi)$ is integrable in a neighborhood of $x$.

3) Let $X$ be a projective variety and $L \rightarrow X$ a nef line bundle. We shall say that $L$ is "good" (see Viehweg [9], page 47) if the numerical dimension. of $L$ equals its Kodaira dimension, i.e. $\kappa(L)=\nu(L)$. Recall the following lemma, (see [16]), which gives a caracterisation of this type of line bundles. 
Lemma (Kawamata). The next two conditions are equivalent:

i) $L$ is nef and good.

ii) There exists a modification $\tau: Z \rightarrow X$, a positive integer $n_{0}$ and an effective divisor $D$ on $Z$ such that

$$
L_{(k)}:=\tau^{*} L^{k} \otimes \mathcal{O}_{Z}(-D)
$$

is semi-ample for all positive $k$ which are divisible by $n_{0}$.

Then the first Chern class of every nef and good line bundle is nef and integrable (for some other properties concerning nef and good bundles, see [17]). Actually, the result of Kawamata implies the semi-positivity of $L_{(k)}$, so for all $k$ divisible by $n_{0}$ there exists a quasi-psh function $\phi_{k}$ on $X$ such that

$$
k \tau^{*} \Theta_{h}(L)+i \partial \bar{\partial} \phi_{k}-[D]=\Theta_{h_{k}}\left(L_{(k)}\right) \geq 0 .
$$

The right hand side of this equality is a smooth $(1,1)$-form, thus the singular part of $\phi_{k}$ does not depend on $k$ (only the "smooth part" of $\phi_{k}$ moves with respect to $k$, as can be easily seen by taking the restriction of (15) to contractible open sets of $X$ ). So one can find $k_{0} \gg 0$ such that $\frac{1}{k_{0}} \max _{Z} \nu\left(\phi_{k_{0}}, x\right)<2$. We denote $\phi:=\frac{1}{k_{0}} \phi_{k_{0}}$. Then inequality (15) implies

$$
\tau^{*} \Theta_{h}(L)+i \partial \bar{\partial} \phi \geq 0
$$

on $Z$; by taking the direct image of $(16)$ we get

$$
\Theta_{h}(L)+i \partial \bar{\partial} \tau_{*} \phi \geq 0
$$

(in the sense of currents on $X$ ). In addition, $\exp \left(-\tau_{*} \phi\right)$ is integrable on $X$, as one can see by performing a change of variables (this is exactly at this point that we need to control the Lelong numbers of $\phi$ ).

Proof of proposition 2.1. As we will see in a moment, the proof is entirely based on the following theorem of J.-P. Demailly, concerning regularisations of closed positive currents (see [5]).

Theorem (Demailly). Let $\varphi$ be a quasi-psh function on $X$ such that $i \partial \bar{\partial} \varphi \geq \gamma$ where $\gamma$ is a smooth (1,1)-form $X$. Then there exists a sequence of functions $\left(\varphi_{m}\right)_{m>0}$ with logarithmic poles on $X$, such that 
1) $\varphi \leq \varphi_{m} \leq \varphi+C$ where $C$ is a constant which does not depend on $m$.

2) $i \partial \bar{\partial} \varphi_{m} \geq \gamma-\delta_{m} \omega$ where $\left(\delta_{m}\right)_{m>0}$ is a sequence of positive numbers which goes to 0 .

The property of integrability of the class $\{\alpha\}$ gives a closed positif current $T=\alpha+i \partial \bar{\partial} \varphi$ with $\exp (-\varphi)$ integrable on $X$; by the previous result of Demailly, we get a sequence of currents $T_{m}:=\alpha+i \partial \bar{\partial} \varphi_{m}$ such that $T_{m} \geq$ $-\frac{1}{m} \omega$. The function $\varphi$ is bounded from above, so we can renormalize $\varphi_{m}$ such that $\varphi_{m} \leq 0$ and still have $\int_{X} \exp \left(-\varphi_{m}\right) d V \leq C$ (independent of $m$ ).

We take by definition $f_{m, 1}:=\varphi_{m}$. The functions $f_{m, 1}$ are smooth on $X$ minus an analytic subset, and they satisfy the assumptions i) and iii) of proposition 2. Now the class $\{\alpha\}$ is nef, so there exists a sequence of smooth functions $\left(f_{m, 2}\right)_{m>0}$ on $X$, such that $\alpha+i \partial \bar{\partial} f_{m, 2} \geq-\frac{1}{m} \omega$ and $\max _{X}\left(f_{m, 2}\right)=0$. With this normalization, we can define for all $m>0$

$$
f_{m}:=\max _{\text {reg }}\left(f_{m, 1}, f_{m, 2}\right)
$$

where $\max _{r e g}$ is ... Then by the standard properties of the regularized maximum function, it is clear that $f_{m}$ is smooth, that its Hessian form is bounded from below by $-\alpha-\frac{1}{m} \omega$ and that $\max _{X}\left(f_{m}\right)=0$. As $-\max (f, g) \leq-f$, the sequence $\left(f_{m}\right)_{m}$ has all the properties required in proposition 2 .

We can now formulate the main result of the second part.

Theorem 2. Let $X$ be a compact Kähler manifold with numerically effective and integrable Ricci class. Then $X$ has a sequence of metrics satisfying all properties stated in theorem 1.

Proof of theorem 2. By our assumption, the Ricci class of $X$ is nef and integrable, so we are in a position to apply proposition 2.1 . In this way we obtain a sequence $\left(f_{k}\right)_{k>0}$ of smooth functions such that

i) $\max _{X} f_{k}=0$.

ii) $\int_{X} \exp \left(-f_{k}\right) d V_{\omega} \leq C$.

iii) $\operatorname{Ricci}_{\omega}+i \partial \bar{\partial} f_{k} \geq-1 / k \omega$ on $X$.

Next, we use precisely these functions to get a sequence of Kähler metrics $\left(\omega_{k}\right)_{k}$ on $X$ as in [7]. With a little bit of anticipation, let us point out that 
it is indeed the property ii) of the sequence $\left(f_{k}\right)_{k}$ which will imply that the volume of the geodesic balls of the Aubin-Calabi-Yau metrics is bounded from below.

Now, we can solve the Monge-Ampère equation (see [7])

$$
\omega_{k}^{n}=\exp \left(1 / k \varphi_{k}-f_{k}\right) \omega^{n}
$$

where $\omega_{k}:=\omega+i \partial \bar{\partial} \varphi_{k}$ and get in this way a sequence of metrics which satisfies the first two assumptions of theorem 1. Thanks to the AubinCalabi-Yau theorem, the solution $\varphi_{k}$ of equation (17) is unique. One can then renormalize this function so that

$$
\int_{X} \varphi_{k} d V_{\omega}=0
$$

and so equation (17) becomes

$$
\omega_{k}^{n}=\lambda_{k} \exp \left(1 / k \varphi_{k}-f_{k}\right) \omega^{n}
$$

where $\lambda_{k}$ is the normalization constant.

The interest of this renormalisation is explained by the following (standard) lemma:

Lemma 2.2. There exists a constant $C_{1}>0$ (which depends only on $(X, \omega))$ such that for each function $\varphi \in \mathcal{C}^{\infty}(X)$ which satisfies $\int_{X} \varphi d V_{\omega}=0$ and $\omega+i \partial \bar{\partial} \varphi \geq 0$, we have: $\sup _{X} \varphi \leq C_{1}$.

(see for example [20]).

Then the new functions, which we still denote by $\varphi_{k}$, have a uniform upper bound, namely $C_{1}$. Now by integrating over $X$ equality (18) we get

$$
\lambda_{k}=\frac{\operatorname{Vol}_{\omega}(X)}{\int_{X} \exp \left(1 / k \varphi_{k}-f_{k}\right) d V_{\omega}} \geq \frac{\operatorname{Vol}_{\omega}(X)}{C \int_{X} \exp \left(-f_{k}\right) d V_{\omega}} \geq C .
$$

In conclusion, $\lambda_{k} \geq C$, uniformly with respect to $k$. We will now show that, thanks to this lower bound for the normalization sequence, we get a lower bound for the volume of certain geodesic balls of $\left(X, \omega_{k}\right)$; in particular, the sequence of volumes cannot go to zero.

For this purpose we recall the next lemma, due to Demailly, Peternell and Schneider, which despites its extremely elementary proof, is one of the main tools in investigating compact Kähler manifolds with nef Ricci class. 
Lemma ([7]). Let $U$ be a compact subset of $\tilde{X}$. For each $\delta>0$, there exist a subset $U_{k, \delta} \subset U$, such that $\operatorname{Vol}_{\omega}\left(U \backslash U_{k, \delta}\right)<\delta$ and such that any two points $x_{1}, x_{2} \in U_{k, \delta}$ can be joined by a path whose length with respect to $\omega_{k}$ is smaller than $C \delta^{-\frac{1}{2}}$, where the constant $C$ is independent of $\delta, k$.

Actually this result is formulated for subsets of universal cover of $X$, but we can equally well apply it for the subsets of $X$-in particular to $X$ himself. So for all $\delta>0$ we get a family of open sets $U_{k, \delta} \subset X$ such that:

1) $\operatorname{Vol}_{\omega}\left(U_{k, \delta}\right) \geq \operatorname{Vol}_{\omega}(X)-\delta$

2) $\operatorname{diam}_{\omega_{k}}\left(U_{k, \delta}\right) \leq C \delta^{-1 / 2}$.

Then for $\delta$ small enough, which will be fixed from now on, we can choose $p_{k} \in U_{k, \delta}$ and in this way we obtain a sequence of geodesic balls $B_{\omega_{k}}\left(p_{k}, C_{1}\right)$ such that

$$
\operatorname{Vol}_{\omega}\left(B_{\omega_{k}}\left(p_{k}, C_{1}\right)\right) \geq \frac{1}{2} \operatorname{Vol}_{\omega}(X) .
$$

On the other hand we have

$$
\int_{B_{\omega_{k}}\left(p_{k}, C_{1}\right)}\left|\varphi_{k}\right| d V_{\omega} \leq 2 C \operatorname{Vol}(X)
$$

where $C$ is the upper bound of $\varphi_{k}$. Consider the set

$$
V_{k}:=\left\{x \in B_{\omega_{k}}\left(p_{k}, C_{1}\right) /\left|\varphi_{k}(x)\right|<8 C\right\} .
$$

By a standard computation, one can see that $\operatorname{Vol}_{\omega}\left(V_{k}\right) \geq 1 / 4 \operatorname{Vol}(X)$ and now we can get a uniform lower bound for the volume of the ball $B_{\omega_{k}}\left(p_{k}, C_{1}\right)$ with respect to $\omega_{k}$. Actually we have:

$$
\begin{aligned}
\operatorname{Vol}_{\omega_{k}}\left(B_{\omega_{k}}\left(p_{k}, C_{1}\right)\right) & =\lambda_{k} \int_{B_{\omega_{k}}\left(p_{k}, C_{1}\right)} \exp \left(1 / k \varphi_{k}-f_{k}\right) d V_{\omega} \\
& \geq C \int_{B_{\omega_{k}}\left(p_{k}, C_{1}\right)} \exp \left(1 / k \varphi_{k}\right) d V_{\omega} \geq C_{3} \operatorname{Vol}_{\omega}\left(V_{k}\right) \\
& \geq C_{4} .
\end{aligned}
$$

This sequence of relations implies (eventually by using the Bishop-Gromov theorem if $C_{1} \geq 1$ ) that for all $k>0$ we have:

$$
\operatorname{Vol}_{\omega_{\varepsilon}}\left(B_{\omega_{\varepsilon}}\left(p_{\varepsilon}, 1\right)\right) \geq C .
$$

We now prove the following lemma, which is well-known in the case of manifolds of semi-positive Ricci curvature: 
Lemma 2.3. Let $(X, g)$ be a m-dimensional complete Riemannian manifold such that the Ricci curvature of $g$ is bounded from below by $-\delta^{2}$. Then for all points $p \in X$ and all radius $1<R<\max \left\{d_{g}(p, q) / q \in X\right\}$, one has the inequality

$$
\frac{\operatorname{Vol}_{g}\left(B_{p}(2 R+2)\right)}{\operatorname{Vol}_{g}\left(B_{p}(1)\right)} \geq \frac{R-1}{2 m+2 \delta(m-1)(R+1)} .
$$

Proof. The proof consists in a slight modification of the arguments given in [23] in the semi-positive case; for the reader's convenience we will sketch it below.

Let $x_{0} \in \partial B_{p}(R)$ (so that $d_{g}\left(p, x_{0}\right)=R$ ); consider the function $\rho(x)=$ $d_{g}\left(x_{0}, x\right)$. It is well known that

$$
\Delta(\rho) \leq \frac{m-1}{\rho}(1+\delta \rho)
$$

in the sense of distributions on $X$ (see for instance [23]; the Laplace operator used here has negative eigenvalues) and also that the norm of the gradient of $\rho$ equals 1 . So we have $\Delta\left(\rho^{2}\right)=2 \rho \Delta(\rho)+2 \leq 2 m+2 \delta(m-1) \rho$, and for every smooth compactly supported positive function $\phi$ on $X$, we get

$$
\int_{X} \phi \Delta\left(\rho^{2}\right) d V \leq 2 m \int_{X} \phi d V+2 \delta(m-1) \int_{X} \rho \phi d V .
$$

By a standard density argument, inequality (21) still holds for every compactly supported Lipschitz function $\phi \geq 0$. Let $\psi:[0, \infty) \rightarrow \mathbb{R}_{+}$be a function with support in $[0, R+1]$, equal to 1 on $[0, R-1]$, such that $\psi(t)=\frac{1}{2}(R-t+1)$ for $\left.\left.t \in\right] R-1, R+1\right]$, and let $\phi(x):=\psi(\rho(x))$. Then $\phi$ is Lipschitz, compactly supported in the ball $B_{x_{0}}(R+1)$, and we have

$$
\begin{aligned}
\int_{X} \phi(x) \Delta\left(\rho^{2}\right)(x) d V_{g} & =-\int_{X} \nabla \phi(x) \nabla \rho^{2}(x) d V_{g} \\
& =-2 \int_{B_{x_{0}}(R+1)} \psi^{\prime}(x)(\rho(x)) \rho(x)|\nabla \rho|^{2}(x) d V_{g} \\
& =\int_{B_{x_{0}}(R+1) \backslash B_{x_{0}}(R-1)} \rho(x) d V_{g} \\
& \geq(R-1)\left(\operatorname{Vol}_{g}\left(B_{x_{0}}(R+1)\right)-\operatorname{Vol}_{g}\left(B_{x_{0}}(R-1)\right)\right) .
\end{aligned}
$$


On the other hand, inequality (21) implies

$$
\begin{aligned}
\int_{X} \phi(x) \Delta\left(\rho^{2}\right)(x) d V_{g} & \leq 2 m \int_{X} \phi(x) d V_{g}+2 \delta(m-1) \int_{B_{x_{0}}(R+1)} \rho(x) d V_{g} \\
& \leq(2 m+2 \delta(m-1)(R+1)) \operatorname{Vol}_{g}\left(B_{x_{0}}(R+1)\right) .
\end{aligned}
$$

By the choice of $x_{0}$ we get $B_{p}(1) \subset B_{x_{0}}(R+1) \backslash B_{x_{0}}(R-1)$ and equally $B_{x_{0}}(R+1) \subset B_{p}(2 R+2)$. So by the previous inequality and these relations we obtain

$$
(2 m+2 \delta(m-1)(R+1)) \operatorname{Vol}_{g}\left(B_{p}(2 R+2)\right) \geq(R-1) \operatorname{Vol}_{g}\left(B_{p}(1)\right),
$$

and we infer the desired estimate.

Now we apply this estimate for our sequence of metrics with $p:=p_{k}$ and $R:=d_{k} / 2$. We get

$$
\frac{d_{k}-2}{4 n+2 / k(2 n-1)\left(d_{k}+2\right)} \leq \frac{\operatorname{Vol}(X)}{\operatorname{Vol}_{\omega_{k}}\left(B_{\omega_{k}}\left(p_{k}, 1\right)\right)}
$$

Inequality (20) shows that the right hand side of this relation has a uniform upper bound $C$, so

$$
\frac{d_{k}-2}{4 n+1 / k(2 n-1)\left(d_{k}+2\right)} \leq C
$$

and it is easily seen that this gives a uniform upper bound for the sequence of diameters $d_{k}$. Theorem 2 is proved.

\section{Remarks.}

1) If we only suppose that $c_{1}(X)$ has property of integrability, the surjectivity of the Albanese map of $X$ was anounced by Demailly-Peternell-Schneider: (see [8]) (which moreover show that the Albanese map is a smooth submersion).

2) For the proof of theorem 2 , we have used a sequence of functions $\left(f_{k}\right)_{k}$ (arising from the fact that the Ricci class of $X$ contains some closed positive current with small Lelong numbers) whose behaviour allows us to get a lower bound for the normalization constant. In general, if $\{\alpha\}$ is a pseudoeffective cohomology class one can get a closed positive current $T \in\{\alpha\}$ with minimal singularities (see [8]). It seems difficult, but perhaps not impossible, to show that by regularizing this current (see [5]) and solving the MongeAmpère equation with the sequence of potentials so obtained, one gets some 
remarkable properties of the sequence of metrics $\omega_{k}$. The major difficulty is to understand the nature of singularity's of this special current $T$.

3) We will now see that in general integrability property of $c_{1}(X)$ is not necessary to have a sequence of metrics for which the demands i)-iii) of theorem 1 are satisfied. For this we revisit an example of Demailly-PeternellSchneider (see [6]).

Let us recall the construction of the variety $X$ they consider. Let $\Gamma:=$ $\mathbb{C} /(\mathbb{Z}+i \mathbb{Z})$ be an elliptic curve and $E \rightarrow \Gamma$ a rank 2 vector bundle given by

$$
E=\mathbb{C} \times \mathbb{C}^{2} /(\mathbb{Z}+i \mathbb{Z}),
$$

where the action of $\mathbb{Z}+i \mathbb{Z}$ is given by the two automorphism

$$
\begin{aligned}
& g_{1}\left(z ; z_{1}, z_{2}\right)=\left(z+1 ; z_{1}, z_{2}\right), \\
& g_{2}\left(z ; z_{1}, z_{2}\right)=\left(z+i ; z_{1}+z_{2}, z_{2}\right) .
\end{aligned}
$$

Finally, let $X$ be the projectivised bundle $\mathbb{P}(E)$ of $E$. In [6], the authors proved that the Ricci class of $X$ is nef, but is not integrable. Here we will indicate explicitly a sequence of Kähler metrics $\left(\omega_{k}\right)$ which are in the same cohomology class, whese Ricci curvature is almost positive, and such that the sequence of diameters has a (finite) upper bound.

Consider the sequence of hermitian metrics on $\mathbb{C}^{2}$, (seen as a trivial bundle on $\mathbb{C}$ ) defined by

$$
\left|\left(z_{1}, z_{2}\right)\right|_{z, k}^{2}:=\left|z_{1}\right|^{2}-\operatorname{Im}(z)\left(z_{1} \bar{z}_{2}+\bar{z}_{1} z_{2}\right)+\left(k+\operatorname{Im}^{2}(z)\right)\left|z_{2}\right|^{2} .
$$

For all $k>4$ we take the differential form

$$
\omega_{k}=i \partial \bar{\partial} \log \left|\left(z_{1}, z_{2}\right)\right|_{z, k}^{2}+i d z \wedge d \bar{z} .
$$

It is well-defined on $X$. A computation without major difficulties (see [19]) shows that

a) for $k>4$, the $(1,1)$ form $\omega_{k}$ is closed and positively defined; it belongs to a fixed cohomology class.

b) the Ricci curvature of $\omega_{\varepsilon}$ is bounded from below by $-1 / k$.

c) the diameter of $X$ with respect to $\omega_{k}$ has a uniform upper bound.

This example indicates that a bound for the sequence of diameters does not necessarily contradict the presence of positive Lelong numbers in the limit current of the sequence of metrics. Actually, our hope is that the following conjecture is true: 
Conjecture. Let $(X, \omega)$ be a compact Kähler manifold, with numerically effective Ricci class. Then $X$ has a sequence of Kähler metrics $\omega_{k}$ such that $\omega_{k} \in\{\omega\}, \operatorname{Ricci}_{\omega_{k}} \geq-1 / k \omega_{k}$ and $d_{k}:=\operatorname{diam}\left(X, \omega_{k}\right)$ has an uniform upper bound.

4) Thanks to the notion of multiplier sheaf of Demailly-Nadel we will now see that we can localize in a precise manner the set $\mathcal{N D}\left(X,\left(\omega_{k}\right)_{k}\right)$, if the sequence of metrics $\omega_{k}$ is obtained by the Monge-Ampère equation (18). For this we have to analyze two cases:

i) The renormalisation sequence $\lambda_{k}$ is bounded from below by a positive constant.

It is then easy to see that $\mathcal{N D}\left(X,\left(\omega_{k}\right)_{k}\right)=X$, by the same type of arguments as those of the second part.

ii) The sequence $\lambda_{k}$ goes to zero.

In this case by inequality (19), we observe that $\int_{X} \exp \left(-f_{k}\right) d V_{\omega} \mapsto \infty$ as $k$ goes to infinity. We put $\tau_{k}:=(1+1 / n) f_{k}$ and let $\mathcal{I}$ be the Demailly-Nadel multiplier ideal associated to $\left(\tau_{k}\right)_{k}$ (see [18]). Consider the variety $\mathcal{V}$ of zeros of this ideal. Then the points of $X \backslash \mathcal{V}$ are characterized in the following manner: $p \in X \backslash \mathcal{V}$ if and only if there exists a neighborhood $W=W(p)$ of this point and $\delta \in] \frac{n}{n+1}, 1\left[\right.$ such that $\int_{W} \exp \left(-\delta \tau_{k}\right) d V_{\omega}<\infty$, (uniformly with respect to $k$ ).

So for each point $p \in X \backslash \mathcal{V}$ we get a neighborhood $W$ such that

$$
\begin{aligned}
\operatorname{Vol}_{\omega_{k}}(W) & =\lambda_{k} \int_{W} \exp \left(1 / k \varphi_{k}-f_{k}\right) d V_{\omega} \leq \lambda_{k} \int_{W} \exp \left(-f_{k}\right) d V_{\omega} \leq \\
& \leq \lambda_{k} \int_{W} \exp \left(-\delta \tau_{k}\right) d V_{\omega} \mapsto 0 .
\end{aligned}
$$

Therefore $p \in \mathcal{D}\left(X,\left(\omega_{k}\right)_{k}\right)$. In conclusion, $\mathcal{N D}\left(X,\left(\omega_{k}\right)_{k}\right) \subset \mathcal{V}$.

3.

Let $X$ be a compact Kähler manifold with numerically effective Ricci class. Then its irregularity $q(X)$ is bounded by the complex dimension of $X$ (see [19]). On the other hand, in the first part of this article we have proved that if the sequence of diameters $d_{k}:=\operatorname{diam}\left(X, \omega_{k}\right)$ is uniformly bounded, the Albanese morphism of $X$ is surjective. In this (last) part we obtain some results in the case $d_{k} \mapsto \infty$. Our result is the following 
Theorem 3. Let $X$ be be a compact complex manifold endowed with a sequence of Kähler metrics $\left(\omega_{k}\right)_{k}$ such that:

i) each metric $\omega_{k}$ belongs to a fixed cohomology class, and Ricci $_{\omega_{k}} \geq$ $-1 / k \omega_{k}$.

ii) There exists a constant $C>0$ and a sequence of positive numbers $\left(C_{k}\right)_{k}$ such that $\lim _{k \rightarrow \infty} C_{k} / \sqrt{k}=0$ and for each $\gamma$ in $H_{1}(X, \mathbb{Z})$ modulo torsion we have:

$$
C \leq|\gamma|_{k, p} \leq C_{k} l_{a l g}(\gamma)
$$

for all $p \in X$.

Then if $d_{k}:=\operatorname{diam}\left(X, \omega_{k}\right)$ goes to infinity, the irregularity of $X$ is bounded by $n-1$.

Before proving this result, we make some remarks. Hypothesis 3.i) is equivalent to the numerical effectivity of the Ricci class. Intuitively, the hypothesis $3 . i i)$ means that the collapsing of the sequence $\left(X, \omega_{k}\right)$ occurs only in the simply-connected part of $X$. Actually, the right hand side of this inequality is always satisfied for certain points $p \in X$; here we suppose that in the other points, the geometrical norm of the non-torsion elements of $H_{1}$ does not go to infinity too fast.

Proof of the theorem 3. The idea of the proof is as follows. In the hypothesis we have made, there exists a renormalisation of the sequence of metrics $\left(\omega_{k}\right)_{k}$ such that the limit in the sense of Gromov-Hausforff of the sequence of pointed varieties $\left(\bar{X}, \bar{g}_{k}, \bar{q}_{k}\right)$ is precisely $\mathbb{R}^{m}$, with $m \leq 2 n-1$ (we use for this some results of Fukaya-Yamaguchi and T. Colding). We denote by $\bar{X}$ the cyclic cover of $X$ and by $\bar{g}_{k}$ the inverse image of the renormalisation of the metric $\omega_{k}$. The next point is to construct (by a standard procedure) an action of $\mathbb{Z}^{b_{1}(X)}$ on the limit space $\mathbb{R}^{m}$, which is discontinuous in zero and faithful. This obviously shows that $b_{1}(X) \leq m$, so we get the conclusion.

Let us fix some notation. Let $h: \pi_{1}(X) \rightarrow H_{1}(X, \mathbb{Z})$ be the Hurewicz morphism and $\Gamma:=h^{-1}(T)$ where $T$ denotes the torsion part of the first homology group. Then we define $\bar{X}:=\widetilde{X} / \Gamma$. The fundamental group of $\bar{X}$ is equal to $\Gamma$ and $\mathbb{Z}^{b_{1}(X)}$ acts on $\bar{X}$ isometrically with respect to any inverse image metric.

We consider the sequence $\left(\bar{X}, \frac{1}{k} \bar{\omega}_{k}, \bar{p}\right)_{k}$ with $\bar{p} \in \bar{X}$ arbitrarily chosen. The Ricci curvature of the metric $\frac{1}{k} \bar{\omega}_{k}$ is bounded from below by -1 . We can then use the following result, due to Fukaya-Yamaguchi. 
Theorem (Fukaya-Yamaguchi). There exists a sequence of real positive numbers $\left(r_{k}\right)_{k}$ which goes to infinity, such that $r_{k} \leq \sqrt{k} / C_{k}$, and a sequence of points $\bar{q}_{k} \in \bar{X}$ such that

$$
\lim _{k \rightarrow \infty}\left(\bar{X}, r_{k} / \sqrt{k} \bar{\omega}_{k}, \bar{q}_{k}\right)=\left(\mathbb{R}^{m}, d, 0\right)
$$

where $d$ is the flat metric on $\mathbb{R}^{m}$.

Let us first say a few words about the proof of this theorem. By possibly passing to a sub-sequence, we can suppose that $\left(\bar{X}, \frac{1}{k} \bar{\omega}_{k}, \bar{p}\right)_{k}$ converges to a length space $\left(Y, d_{Y}, q\right)$. If $Y$ is not reduced to a point, let $z \neq y$ and let $y_{1}$ a point located in the interior of the minimizing geodesic between $y$ et $z$. Consider the sequence of pointed spaces $\left(Y, r_{k} d_{Y}, z\right)_{k}$ where $\left(r_{k}\right)_{k}$ is a sequence of positive numbers which can be chosen to go at infinity as slow as we like. Then we can get a line in the limit space by rescaling the geodesic between $y$ and $z$. The splitting theorem of Cheeger-Colding (see [1]) shows that the limit space $Y_{1}$ is isometric to $\mathbb{R} \times Y_{2}$. But $Y_{1}$ is also the limit of the sequence $\left(\bar{X}, \frac{r_{k}}{\sqrt{k}} d_{k}, \bar{q}_{k}\right)$ (if $r_{k}$ goes to infinity slow enough). By iterating this construction at most $2 n$ times, one obtains a proof of the above theorem. We mention here another result of Cheeger- Colding which says that actually the points of $Y$ where the tangent cone is isometric to a Euclidean space are dense in $Y$ (see [2]).

Next, we want to get an upper bound for $m$; in general, one can only have $m \leq 2 n$, but in our (special) situation we prove that $m \leq 2 n-1$. To see this, observe first that if the sequence of diameters $d_{k}$ goes to infinity, then for all family of points $q_{k} \in X$, the volume of the geodesic balls of radius 1 , centered at $q_{k}$ of $\left(X, \omega_{k}\right)$, goes to zero. We use again lemma 2.3, applied to the following data: the metric $g$ equals $\omega_{k}, p:=q_{k}$ and $R=\frac{1}{2} \operatorname{diam}\left(X, \omega_{k}\right)$. In this way we get

$$
\frac{\operatorname{Vol}_{\omega_{k}}(X)}{\operatorname{Vol}_{\omega_{k}}\left(B_{\omega_{k}}\left(q_{k}, 1\right)\right)} \geq \frac{d_{k}-2}{4 n+1 / \sqrt{k}(2 n-1)\left(d_{k}+2\right)}
$$

thus

$$
\operatorname{Vol}_{\omega_{k}}\left(B_{\omega_{k}}\left(q_{k}, 1\right)\right) \leq\left(\frac{4 n}{d_{k}-2}+\frac{1}{\sqrt{k}} \frac{d_{k}+2}{d_{k}-2}\right) \operatorname{Vol}(X)
$$

and it is obvious that the right hand side of the above inequality goes to zero. Now, thanks to the fact that the geometrical norm of each free element of $H_{1}(X, \mathbb{Z})$ is uniformly bounded from below, we obtain

$$
\operatorname{Vol}_{\bar{\omega}_{k}}\left(B_{\bar{\omega}_{k}}\left(\bar{q}_{k}, C\right)\right)=\operatorname{Vol}_{\omega_{k}}\left(B_{\omega_{k}}\left(q_{k}, C\right)\right)
$$


for some constant $C>0$, where $q_{k}$ is the projection of $\bar{q}_{k}$ on $X$. If the constant $C$ is smaller than 1 , we see that $\lim _{k \rightarrow \infty} \operatorname{Vol}_{\bar{\omega}_{k}}\left(B_{\bar{\omega}_{k}}\left(\bar{q}_{k}, C\right)\right)=0$. By the Bishop-Gromov inequality we obtain

$$
\frac{\operatorname{Vol}_{\bar{\omega}_{k}}\left(B_{\bar{\omega}_{k}}\left(\bar{q}_{k}, 1\right)\right)}{\operatorname{Vol}_{\bar{\omega}_{k}}\left(B_{\bar{\omega}_{k}}\left(\bar{q}_{k}, C\right)\right)} \leq C_{1}
$$

and so $\lim _{k \rightarrow \infty} \operatorname{Vol}_{\bar{\omega}_{k}}\left(B_{\bar{\omega}_{k}}\left(\bar{q}_{k}, 1\right)\right)=0$. The case $C>1$ gives a similar conclusion. In the following lines, we will see that this result still holds for the renormalized metrics $\bar{g}_{k}:=\frac{r_{k}^{2}}{k} \bar{\omega}_{k}$. Actually we have

$$
\operatorname{Vol}_{\bar{g}_{k}}\left(B_{\bar{g}_{k}}\left(\bar{q}_{k}, 1\right)\right)=\frac{r_{k}^{2 n}}{k^{n}} \operatorname{Vol}_{\bar{\omega}_{k}}\left(B_{\bar{\omega}_{k}}\left(\bar{q}_{k}, \frac{\sqrt{k}}{r_{k}}\right)\right)
$$

and by the Bishop-Gromov inequality once again we get

$$
\begin{aligned}
\operatorname{Vol}_{\bar{\omega}_{k}}\left(B_{\bar{\omega}_{k}}\left(\bar{q}_{k}, \frac{\sqrt{k}}{r_{k}}\right)\right) & \leq \frac{\int_{0}^{\frac{1}{r_{k}}} s h^{2 n-1} t d t}{\int_{0}^{\frac{1}{\sqrt{k}}} s h^{2 n-1} t d t} \operatorname{Vol}_{\bar{\omega}_{k}}\left(B_{\bar{\omega}_{k}}\left(\bar{q}_{k}, 1\right)\right) \\
& \leq C \frac{k^{n}}{r_{k}^{2 n}} \operatorname{Vol}_{\bar{\omega}_{k}}\left(B_{\bar{\omega}_{k}}\left(\bar{q}_{k}, 1\right)\right)
\end{aligned}
$$

because the sequence $r_{k}$ goes to infinity. Hence, with respect to the metrics $\bar{g}_{k}$, we have

$$
\operatorname{Vol}_{\bar{g}_{k}}\left(B_{\bar{g}_{k}}\left(\bar{q}_{k}, 1\right)\right) \leq C \operatorname{Vol}_{\bar{\omega}_{k}}\left(B_{\bar{\omega}_{k}}\left(\bar{q}_{k}, 1\right)\right)
$$

and so the volume of the geodesic balls of center $\bar{q}_{k}$, and radius 1 of the manifold $\left(\bar{X}, \bar{g}_{k}\right)$ goes to zero. The interest of this observation is explained by the next theorem, due to Cheeger-Colding (see [2]).

Theorem (Cheeger-Colding). Let $\left(M_{k}, g_{k}, q_{k}\right)_{k}$ a sequence of m-dimensional complete Riemannian manifolds, such that

$$
\operatorname{Vol}_{g_{k}}\left(B_{g_{k}}\left(q_{k}, 1\right)\right) \rightarrow 0 \text { and } \operatorname{Ricci}_{g_{k}} \geq-g_{k} .
$$

Then the Hausdorff dimension of (any) limit space is at most equal to $m-1$.

We apply this result to $\left(\bar{X}, \bar{g}_{k}, \bar{q}_{k}\right)$; by the Cheeger-Colding result the sequence converges to a Euclidean space of dimension at most $2 n-1$.

In the final step of the proof we construct a monomorphism $\mathbb{Z}^{b_{1}(X)} \mapsto$ $\operatorname{Isom}\left(\mathbb{R}^{m}\right)$. 
We denote by $d_{g_{k}}$ the distance associated with the metric $\bar{g}_{k}$; then, by the properties of the sequence $\left(r_{k}\right)_{k}$ and the hypothesis 3.ii) of the theorem, we get $d_{g_{k}}\left(\bar{q}_{k}, \gamma_{j} \bar{q}_{k}\right) \leq 1$ for the generators $\gamma_{j}$ of $H_{1}(X, \mathbb{Z})$ modulo torsion. By a procedure due to Gromov (see [13]), for each positive $k$ one can find a sub-group of finite index $\Gamma_{k}$ in $H_{1}(X, \mathbb{Z}) / T$ generated by $\gamma_{1, k}, \cdots \gamma_{b_{1}, k}$ such that:

$$
d_{g_{k}}\left(\bar{q}_{k}, \gamma_{j, k} \bar{q}_{k}\right) \leq 4 \text { and } d_{g_{k}}\left(\bar{q}_{k}, \gamma \bar{q}_{k}\right) \geq 2
$$

for each non-zero $\gamma \in \Gamma_{k}$. Then by Gromov's isometry lemma (see [14]) one can construct

$$
\Psi: \mathbb{Z}^{b_{1}(X)} \rightarrow \operatorname{Isom}\left(\mathbb{R}^{m}\right)
$$

by associating to each generator $\rho_{j}$ of $\mathbb{Z}^{b_{1}(X)}$ the limit (of a subsequence) of the isometries sequence $\gamma_{j, k}$ of $\left(\bar{X}, \bar{g}_{k}\right)$. The fact that the geometric norm at $\bar{q}_{k}$ of each non-trivial element of $\Gamma_{k}$ is bounded by 2 implies the injectivity of $\Psi$ and also the fact that the orbit of the image of this morphism is discontinuous at zero.

In conclusion, we get a free Abelian group of isometries of $\mathbb{R}^{m}$ (endowed with the flat metric) which we denote by $\Gamma_{\infty}$, generated by $\gamma_{1, \infty}, \cdots \gamma_{b_{1}(X), \infty}$ such that: $\left\|\gamma_{j, \infty}(0)\right\| \leq 5$ and $\|\gamma(0)\| \geq 1$ for all $\gamma \neq I d$. But this easily implies $b_{1}(X) \leq m$ (by estimating the intersection of the orbit of $\Gamma_{\infty}$ with balls of large radius). The proof of theorem 3 is complete.

Remark. One can get the same conclusion in theorem 3 by replaccing the hypothesis $|\gamma|_{k, p} \geq C$ with the weaker condition

$$
N_{p}(k, 1) \max \left\{1 / D_{k}, 1 / \sqrt{k}\right\} \rightarrow 0,
$$

where $N_{p}(k, 1)$ is the cardinal of the elements $\gamma \in H_{1}(X, \mathbb{Z})$ such that $d_{\omega_{k}}(\bar{p}, \gamma \bar{p}) \leq 1$. Actually, up to some constants, the volume of the geodesic ball $B_{\bar{\omega}_{k}}(\bar{p}, 1)$ is bounded by the above expression.

\section{Application.}

As a corollary of theorem 3 we have:

Corollary 3.1. Let $X$ be a compact complex manifold as in theorem 3. Then if $b_{1}(X)=2 n$, the manifold $X$ is homeomorphic to a torus.

Proof. By theorem 3, the sequence of diameters $d_{k}=\operatorname{diam}\left(X, \omega_{k}\right)$ is uniformly bounded. So $\left(d_{k} / \sqrt{k}\right)_{k}$ goes to zero, and by the Colding's result below (see [3]), we get the corollary. 
Theorem (Colding). There exists a constant $\delta(m)$ such that every $m-$ dimensional compact Riemannian manifold $(M, g)$ satisfying Ricci $_{g} \geq$ $-\delta(m) g$ and $b_{1}(M)=m$ is homeomorphic to a torus.

\section{References.}

[1] J. Cheeger and T. Colding, Lower bounds on Ricci curvature and almost rigidity of warped products, Ann. Math. 144 (1996), 189-237.

[2] J. Cheeger and T.H. Colding, On the structure of spaces with Ricci curvature bounded below; 1, J. Diff. Geom. 43 (1997).

[3] T.H. Colding, Ricci curvature and volume convergence, Ann. Math. 145 (1997).

[4] J.-P. Demailly, Cohomology of q-convex spaces in top degrees, Math. Z. 204 (1990), 283-295

[5] J.P. Demailly, Regularization of closed positive currents and intersection theory, J. Algebraic Geometry, 1 (1992), 361-409

[6] J.-P. Demailly, Th. Peternell, and M. Schneider, Compact complex manifolds with numerically effective tangent bundles, Journal of Algebraic Geometry, 3 (1994), 295-345.

[7] J.-P. Demailly, Th. Peternell, and M. Schneider, Kähler manifolds with numerically effective Ricci class, Comp. Math. 89 (1993), 217-240.

[8] J.-P. Demailly, Th. Peternell, and M. Schneider, Pseudo-efective line bundles, in preparation.

[9] H. Esnault and E. Viehweg, Lectures on vanishing theorems, DMV Seminar (1992) Band 20.

[10] K. Fukaya and T. Yamaguchi, The fundamental group of almost nonnegatively curved manifolds, Ann. of Math. 136 (1992).

[11] S. Gallot, A Sobolev inequality and some geometric applications, Spectra of riemannian manifolds, Kaigai Publications, Tokio, 1983.

[12] S. Gallot, Volumes, courbure de Ricci et convergence des variétés (d'après T.H. Colding et Cheeger-Colding, Exposé 835, Séminaire Bourbaki, vol. 1997-1998.

[13] M. Gromov, Structures métriques pour les variétés riemanniennes, Cours rédigé par J. Lafontaine et $\mathrm{P}$. Pansu, Textes Mathématiques, 1. Paris: Cedic/Fernand Nathan, Vol. VII, (1981), 152 p. 
[14] M. Gromov, Groups of polynomial growth and expanding maps, Appendix by J. Tits, Publ. I.H.E.S. 53 (1981).

[15] H. Hess, R. Schrader, D.A. Uhlenbrock, Kato's inequality and the spectral distribution of laplacians on compact riemannian manifolds, J. Diff. Geom. bf 15 (1980), 27-37.

[16] Y. Kawamata, Pluricanonical systems on minimal algebraic varieties, Invent. Math. 79 (1985).

[17] C. Mourougane, Thèse, Université de Grenoble 1, Janvier 1997.

[18] A.M. Nadel, Multiplier ideal sheaves and Kähler-Einstein metrics of positive scalar curvature, Ann. Math. 132 (1990), 189-237.

[19] M. Paun, Thèse, Université de Grenoble 1, Janvier 1998.

[20] Séminaire Palaiseau, Première classe de Chern et courbure de Ricci: preuve de la conjecture de Calabi, Astèrisque 58 (1978).

[21] Th. Peternell and F. Serrano, Threefolds with nef anticanonical bundle, to appear in Collectanea mathematica 1998.

[22] H. Skoda, Sous-ensembles analytiques d'ordre fini ou infini dans $\mathbb{C}^{n}$, Bull. Soc. Math. France 100 (1972), 353-408.

[23] R. Schoen and S.-T. Yau, Lectures on differential geometry, Conference proceedings and lecture notes in geometry and topology, edited by N. Hitchin, R. Kirbi, J. Wolf.

[24] Zhang Qi, On projective manifolds with nef anticanonical bundle, J. für die reine und angewandte Math. 478 (1996), 57-60.

RECEIVED OCTOBER 9, 1998.

Université Louis Pasteur

7 RUE REnÉ Descartes

67084 STRAsbourg, France

E-mail address: paun@irma.u-strsbg.fr 